\title{
ALTERACCÕES NO CANAL PRINCIPAL DA BACIA HIDROGRÁFICA DO ARROIO MICHAELA, PELOTAS-RS, DECORRENTES DE UM EVENTO PLUVIOMÉTRICO EXTREMO DO ANO DE 2009
}

\author{
Henrique Noguez da Cunha \\ Universidade Federal de Pelotas - UFPel \\ henriquencunha@gmail.com \\ Adalberto Koiti Miura \\ Embrapa Clima Temperado - CPACT \\ adalberto.miura@embrapa.br \\ Jose Maria Filippini Alba \\ Embrapa Clima Temperado - CPACT \\ jose.filippini@embrapa.br \\ Mauro Ricardo Roxo Nóbrega \\ Fundação Cearense de Meteorologia e Recursos Hídricos - Funceme \\ nobrega.mauro@gmail.com \\ Adriano Luís Heck Simon \\ Universidade Federal de Pelotas - UFPel \\ adrianosimon@gmail.com
}

\begin{abstract}
RESUMO
Eventos extremos de precipitação podem ser responsáveis por reajustes nos elementos naturais de bacias hidrográficas devido à entrada e circulação de matéria e energia excessiva em curtos intervalos de tempo. Este trabalho foi desenvolvido com o objetivo de identificar a extensão das mudanças ocorridas no compartimento de fundo de vale atrelado à zona de vegetação ripária do canal principal da bacia hidrográfica do Arroio Michaela (Pelotas/RS), em função da significativa remoção, transporte e deposição de materiais resultantes do evento pluviométrico extremo ocorrido em janeiro de 2009. Foi elaborada uma classificação sobre o conjunto de imagens diferenças, dos cenários anterior e posterior ao evento, dos Índices de Vegetação, primeira componente principal e imagens-fração sobre imagens Landsat5TM. Os resultados foram superestimados quando utilizada apenas a imagem diferença dos índices de vegetação. A classificação das imagens diferenças dos índices de vegetação com a primeira componente principal e imagens-fração apresentou eliminação dessas incoerências. Os danos foram identificados pela remoção de materiais do terço superior declivoso, remoção e deposição no terço médio sinuoso e deposição no terço inferior plano. Este estudo revela a avaliação do comportamento da rede de drenagem em situações extremas como importante ferramenta de tomada de decisão para uso e ocupação.
\end{abstract}

Palavras-chave: Mudanças Ambientais. Sistemas Ambientais. Sensoriamento Remoto.

\section{CHANGES IN THE MAIN CHANNEL OF THE ARROIO MICHAELA RIVER BASIN, PELOTAS-RS, BRAZIL, RESULTING FROM EXTREME RAINFALL EVENT IN 2009}

\begin{abstract}
Extreme events of rainfall may be responsible for readjustments in the natural elements of river basins due to inward and circulation of matter and excessive energy in short time periods. This work was carried out with the purpose of identifying the extension of changes which occurred in the compartment on the valley floor linked to the riparian vegetation zone of the main channel of the Arroio Michaela river basin (Pelotas/RS), as a result of significant removal, transport and deposition of materials resulting from the extreme rainfall event from January 2009. A classification on the group of difference images, of the scenery before and after the event, the vegetation index, first main component and fraction-images on
\end{abstract}


Alterações no canal principal da bacia hidrográfica do Arroio Michaela, Pelotas-RS, decorrentes de um evento pluviométrico extremo do ano de 2009
Henrique Noguez da Cunha Adalberto Koiti Miura Jose Maria Filippini Alba Mauro Ricardo Roxo Nóbrega Adriano Luís Heck Simon

Landsat5TM images was elaborated. The results were overestimated when only the difference image of vegetation index was used. The classification of the difference images of the vegetation index with the first main component and the fraction-images presented an elimination of these inconsistencies. The damages were identified by the removals of materials of the steep superior third, removal and deposition in the sinuous medium third and deposition in the flat lower third. This study reveals the assessment of behavior of the drainage system in extreme situations as an important tool of decision-making for use and occupancy.

Keywords: Environmental changes. Environmental systems. Remote Sensing.

\section{INTRODUÇÃO}

No final do mês de janeiro do ano de 2009 um evento pluviométrico extremo atingiu o município de Pelotas causando inundações, prejuízos materiais e mortalidades (Schneider, 2009). Este fenômeno climático foi atribuído aos Sistemas Convectivos de Mesoescala (SCM), que costumam ocorrer no Estado do Rio Grande do Sul, principalmente durante os meses quentes de verão e são responsáveis por condições de tempo mais adversas em superfície (granizo, rajadas de ventos, descargas elétricas atmosféricas e precipitações intensas, por exemplo), sempre causando significativas perdas à população e aos setores público e privado (MACHADO et al., 1998; ANABOR, 2004).

Segundo o IPCC (2012), um extremo climático ocorre quando uma variável meteorológica ou climática excede os valores limites (superiores ou inferiores) normalmente observáveis. Marengo (2009) define evento extremo em termos meteorológicos e climatológicos como grandes desvios de um estado climático moderado.

Muito embora incidentes pluviométricos extremos sejam recorrentes na região sul do Brasil (ANABOR, 2004; SAITO et al., 2008; BARBIERI, 2009), as consequências destes eventos podem trazer uma série de alterações aos elementos dos sistemas hidrográficos que recebem cargas extremas de precipitação, atuando na reconfiguração espacial do sistema de drenagem em escala local, trazendo prejuízos às práticas econômicas desenvolvidas no contexto das bacias de captação envolvidas. Esta situação tende a se acentuar em sistemas onde mecanismos de controle diretos e indiretos (DREW, 1983) resultantes das práticas de uso da terra e de obras de engenharia, causam interferências na dinâmica dos elementos naturais (rede de drenagem, escoamento superficial e subsuperficial).

A análise das alterações causadas por eventos extremos em sistemas hidrográficos é uma das ferramentas mais eficientes para a identificação de áreas-chave para a dinâmica dos elementos da bacia hidrográfica em períodos de máxima entrada e circulação de matéria e energia, e que, portanto, precisam ser preservadas do processo de ocupação e melhor entendidas antes da consolidação de obras de engenharia como pontes, estradas e reservatórios. Uma ferramenta importante para análises desta natureza é a utilização de técnicas de sensoriamento remoto, já utilizadas por autores como Venturieri et al (2005) e Abdon et al (2009) para detecção de alterações da dinâmica terrestre.

De acordo com Jensen (2009), o uso de dados obtidos por satélites é uma das formas mais eficazes e econômicas de avaliar alguns aspectos físicos e admite relacionar e modelar dados da dinâmica terrestre, tanto no meio construído, quanto natural. O uso de informações advindas de sensores remotos pode ainda substituir a falta ou carência de dados climáticos e do meio físico, permitindo a implementação de medidas no manejo e no controle das inundações.

Neste sentido, este trabalho tem como objetivo identificar a extensão das mudanças ocorridas no compartimento de fundo de vale atrelado à zona de vegetação ripária do canal principal da bacia hidrográfica $(\mathrm{BH})$ do Arroio Michaela (Pelotas - Rio Grande do Sul), em função da significativa remoção, transporte e deposição de materiais resultantes do evento pluviométrico extremo ocorrido em janeiro de 2009. 
Alterações no canal principal da bacia hidrográfica do Arroio Michaela, Pelotas-RS, decorrentes de um evento pluviométrico extremo do ano de 2009
Henrique Noguez da Cunha Adalberto Koiti Miura Jose Maria Filippini Alba Mauro Ricardo Roxo Nóbrega Adriano Luís Heck Simon

\section{METODOLOGIA}

A bacia hidrográfica do Arroio Michaela está localizada no município de Pelotas, região sul do estado do Rio Grande do Sul, entre as coordenadas 52 $32^{\prime} 37^{\prime \prime} \mathrm{W}, 31^{\circ} 36^{\prime} 34^{\prime \prime S}$ e $52^{\circ} 28^{\prime} 43^{\prime \prime} \mathrm{W}, 31^{\circ} 42^{\prime} 28^{\prime \prime S}$ (Figura 1) e possui uma área de $37,2 \mathrm{~km}^{2}$, tendo sido delimitada por Cunha et al. (2012). Os autores citados também destacaram as principais características morfométricas desta unidade: perímetro de 35,63km, desnível total do talvegue de $220 \mathrm{~m}$, comprimento do talvegue principal de $15,6 \mathrm{~km}$ e comprimento total dos cursos de água de $46,16 \mathrm{~km}$.

Segundo Philip et al. (2002), a litologia da BH é composta predominantemente por rochas da suíte granítica Pinheiro Machado em seus dois terços superiores, estando associada a um relevo com maiores altitudes e declividades, que determina uma maior velocidade de escoamento superficial laminar e linear. Em seu terço inferior verifica-se a ocorrência de litologias sedimentares vinculadas à Formação Graxaim. De acordo com dados do RADAMBRASIL (BRASIL, 1986) e de Delaney (1965) a Formação Graxaim é mal consolidada e apresenta profundidades de aproximadamente 10 metros, sustentando formas do relevo onduladas à planas com presença de depósitos aluviais holocênicos e pouca competência de transporte de materiais por parte dos segmentos de canais fluviais. A localização da $\mathrm{BH}$ em importante zona de transição de compartimentos geológicos e geomorfológicos interfere diretamente no arranjo espacial da rede de drenagem e na dinâmica dos canais fluviais (Figura 1).

Figura 1: Localização da BH do Arroio Michaela, Município de Pelotas-RS.

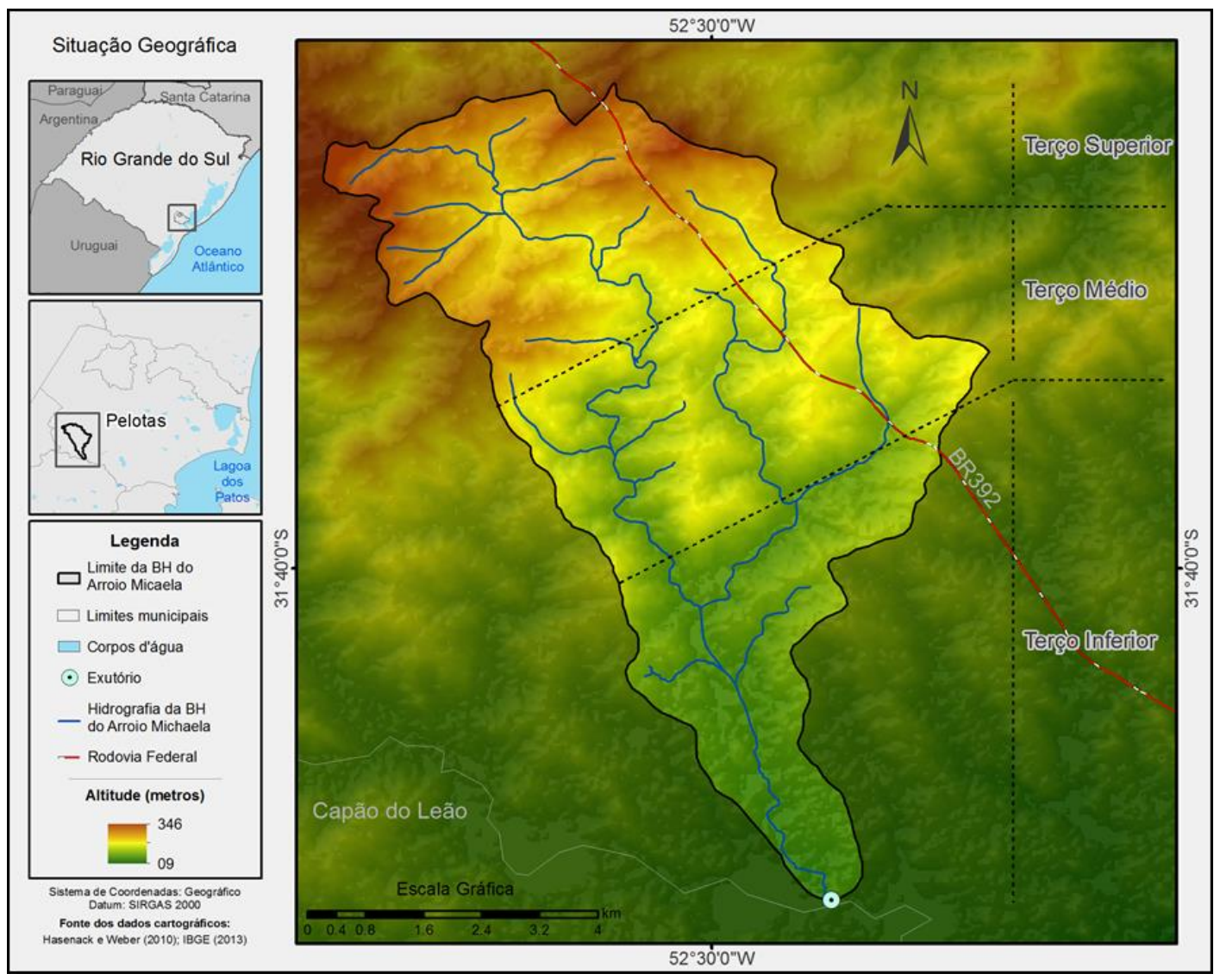

Elaboração: o autor

As informações utilizadas para a identificação e análise das mudanças ocorridas no canal principal da bacia hidrográfica $(\mathrm{BH})$ do Arroio Michaela foram obtidas por meio de dois conjuntos de imagens Landsat5 
Alterações no canal principal da bacia hidrográfica do Arroio Michaela, Pelotas-RS, decorrentes de um evento pluviométrico extremo do ano de 2009
Henrique Noguez da Cunha Adalberto Koiti Miura Jose Maria Filippini Alba Mauro Ricardo Roxo Nóbrega Adriano Luís Heck Simon

TM (WRS 221/82), provenientes do acervo do Instituto Nacional de Pesquisas Espaciais - INPE (http://www.dgi.inpe.br/CDSR), datadas respectivamente de 07.01.2009 e 13.04.2009, períodos anterior e posterior ao evento pluviométrico extremo ocorrido entre os dias 28 e 29 de janeiro de 2009. Concomitantemente foram utilizados os planos de informações (PIs) de rede viária e hidrografia, digitalizados de cartas topográficas do exército, em escala 1:50.000, por Hasenack; Weber (2010).

Todos os procedimentos de processamento digital de imagens e de geoprocessamento foram conduzidos com os softwares ERDAS Imagine® 8.7 (LEICA, 2003) e ArcGis® 9.3 (ESRI, 2008). Inicialmente, as imagens foram registradas com base em uma cena Landsat7 ETM ortorretificada, proveniente do acervo do Global Land Cover Facility (USGS, 2012).

As imagens foram transformadas em valores de reflectância (CHANDER et al., 2009) e submetidas a uma correção atmosférica, com base na subtração do pixel escuro (CHAVEZ, 1988), para minimizar os efeitos de espalhamento, absorção e refração da energia eletromagnética, principalmente para as bandas do visível. Esse pré-processamento permitiu compatibilizar os dados multitemporais, uniformizando-os na mesma escala radiométrica (SONG, et al. 2001; LU et al, 2004).

A partir dos valores de reflectância de superfície obtidos foram calculados, para os dois cenários, os Índices de Vegetação por Diferença Normalizada (NDVI), por meio de operações matemáticas de bandas, utilizando-se a equação proposta por Rouse et al. (1973) (Figura 2). Esse procedimento resulta na identificação de áreas vegetadas e não vegetadas e tem sido utilizado com sucesso para medir e monitorar o vigor da cobertura vegetal a partir das relações entre: a) os altos valores de NDVI e a absorção dos comprimentos de onda do visível por pigmentos envolvidos na fotossíntese; e b) os baixos valores de NDVI com feições não vegetadas, solo exposto, afloramentos rochosos, depósitos aluviais, corpos de água, etc. (TUCKER, 1979; TUCKER et al 1991).

Esse procedimento de discriminação entre as feições vegetadas e não vegetadas, conduzido em uma análise temporal pela técnica de imagem diferença, revela a dinâmica de incorporações e remoções da cobertura vegetal resultantes da ação antrópica ou resultantes da dinâmica natural, como um evento pluviométrico extremo. A partir disso, o índice de vegetação calculado para o primeiro cenário foi subtraído pelo NDVI do segundo cenário, obtendo a imagem diferença do NDVI (Figura 2a), com informações das alterações da cobertura vegetal no intervalo estudado.

A imagem diferença do NDVI foi classificada com base no conjunto de atributos com menor correlação, ou seja, aqueles que expressam as alterações decorrentes da variação temporal. Para isso foi realizada uma classificação supervisionada pelo método da máxima verossimilhança (STRAHLER, 1980), na qual as amostras de treinamento dos principais alvos (corpos de água, cobertura florestal, campestre, áreas agrícolas e alterações decorrentes da inundação), apoiadas por interpretação visual da imagem sobre composição de cores naturais, foram utilizadas como formação das categorias para a classificação (MIURA et al., 2009).

Lu et al. (2004), observam que a avaliação temporal dos índices de vegetação, apesar de identificar a dinâmica ocorrida, tende a realçar ruídos diversos, resultando em incoerências e superestimação dos resultados. Thiam (1997) alerta que a imagem diferença do NDVI pode apresentar problemas na distinção entre uma real alteração e a variabilidade natural, como estiagens sazonais ou mudança no uso da terra.

Nesse sentido, o resultado obtido a partir da classificação da imagem diferença do NDVI foi avaliado por interpretação visual em tela. Apesar de apresentar uma boa separabilidade de classes na análise visual, apresentou certa ambiguidade entre os alvos no processo de classificação digital, isto é, não discriminou corretamente as alterações ocorridas nas margens do Arroio Michaela, comissionando áreas de outras classes (campos, terras em preparo para cultivo, etc.), superestimando o resultado.

Esta situação motivou o aprimoramento dos procedimentos metodológicos, em busca de uma abordagem que minimizasse as incertezas (Figura 2 - quadrado tracejado). Optou-se por complementar a análise incluindo a imagem de componentes principais (CP), utilizados com sucesso por Gong (1993), que reduzem a dimensionalidade dos dados e permitem, a partir das imagens componentes, uma melhor separabilidade de alvos (JOHNSON; WICHERN, 1998) e as imagensfração (solo, vegetação e sombra) derivadas da análise do modelo linear de mistura espectral (MLME) (SHIMABUKURO; SMITH, 1991) conforme sugeridos por Adams et al (1995) e Roberts et al (2002) em trabalhos de deteç̧ão de mudanças na cobertura da terra na Amazônia. 
Alterações no canal principal da bacia hidrográfica do Arroio Michaela, Pelotas-RS, decorrentes de um evento pluviométrico extremo do ano de 2009
Henrique Noguez da Cunha Adalberto Koiti Miura Jose Maria Filippini Alba Mauro Ricardo Roxo Nóbrega Adriano Luís Heck Simon

Figura 2: Fluxograma do processo metodológico de identificação das alterações decorrentes do evento pluviométrico extremo.

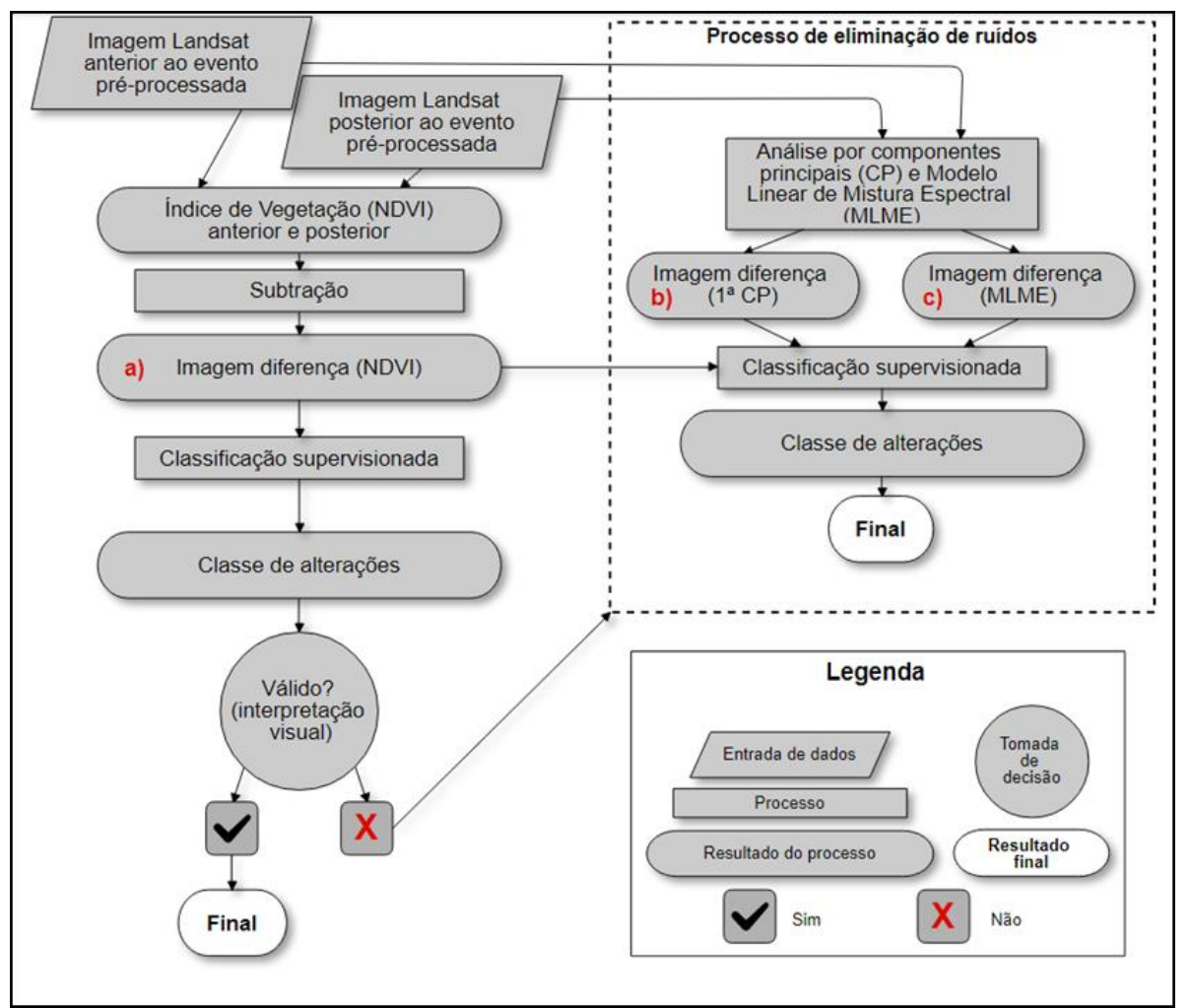

Elaboração: O autor

A técnica do MLME parte do princípio de que diferentes alvos estão integrados dentro do campo de visada do sensor remoto e que um pixel de imagem pode apresentar diferentes percentuais destes mesmos alvos. Segundo Shimabukuro et al. (1998), esta mistura pode ser desfeita em uma relação linear na qual os principais elementos contidos na imagem são separados em imagens-frações às quais contém a proporção dos elementos das imagens de origem, cuja soma deve ser igual a 1. Neste trabalho a seleção dos endmenbers (componentes puros), foi realizada segundo o método proposto por Bateson; Curtiss (1996) para a seleção manual de endmembers, coadjuvado pelas componentes principais, conforme indicações de Muchoney; Haack (1994).

Os planos de informações da primeira componente principal e imagens-fração, dos dois cenários, foram submetidos à técnica de imagem diferença. Em seguida, as imagens-diferença NDVI (Figura 2a), primeira componente principal (Figura 2b) e imagens-fração (MLME) (Figura 2c) foram agrupadas e classificadas pelo método supervisionado de máxima verossimilhança, de forma análoga ao processo de classificação utilizado anteriormente.

A classe de alterações decorrentes da inundação foi vetorizada e exportada para ambiente SIG onde foram relacionadas espacialmente com o plano de informação de rede hidrográfica e com o perfil longitudinal do canal principal.

\section{RESULTADOS E DISCUSSÃO}

O evento pluviométrico de janeiro de 2009 superou os valores médios $(153,3 \mathrm{~mm})$ e a máxima de precipitação registrada no mês de janeiro em $24 \mathrm{~h}(188,2 \mathrm{~mm}$ ) entre os anos de 1971 e 2000 (normais climatológicas - EMBRAPA, 2013). De acordo com Saldanha et al. (2012), o evento ocorrido foi

$\begin{array}{llllll}\text { Caminhos de Geografia } & \text { Uberlândia - MG } & \text { v. 19, n. } 68 & \text { Dez/2018 } & \text { p. 143-156 } & \text { Página } 147\end{array}$


Alterações no canal principal da bacia hidrográfica do Arroio Michaela, Pelotas-RS, decorrentes de um evento pluviométrico extremo do ano de 2009
Henrique Noguez da Cunha Adalberto Koiti Miura Jose Maria Filippini Alba Mauro Ricardo Roxo Nóbrega Adriano Luís Heck Simon

extremo, com um recorde de medição de precipitação no Brasil no período de 24 horas e menores durações. As precipitações ocorridas atingiram a marca de $329 \mathrm{~mm}$ em apenas 3 horas e $586 \mathrm{~mm}$ em 24 horas (EMBRAPA, 2013) superando em mais de três vezes a chuva com tempo de 100 anos de retorno $(98,2 \mathrm{~mm})$.

Esta excepcionalidade teve efeitos diretos sobre a rede de drenagem da $\mathrm{BH}$ do Arroio Michaela, sobretudo ao longo do canal principal, que suportou todo o fluxo trazido pelos canais afluentes desde as cabeceiras de drenagem até a foz, procurando reajustar sua capacidade de transporte a partir de modificações nas margens que subtraíram porções significativas de cobertura vegetal ripária e remobilizaram material rochoso de diferentes tamanhos. O resultado das modificações ocorridas ao longo do perfil longitudinal do Arroio Michaela, evidenciado por meio dos Índices de Vegetação por Diferença Normalizada está apresentado na Figura 3.

Figura 3: a) NDVI da data anterior ao evento (07-01-2009), b) NDVI da data posterior ao evento (1304-2009, c) Imagem diferença entre os índices de vegetação (NDVI).

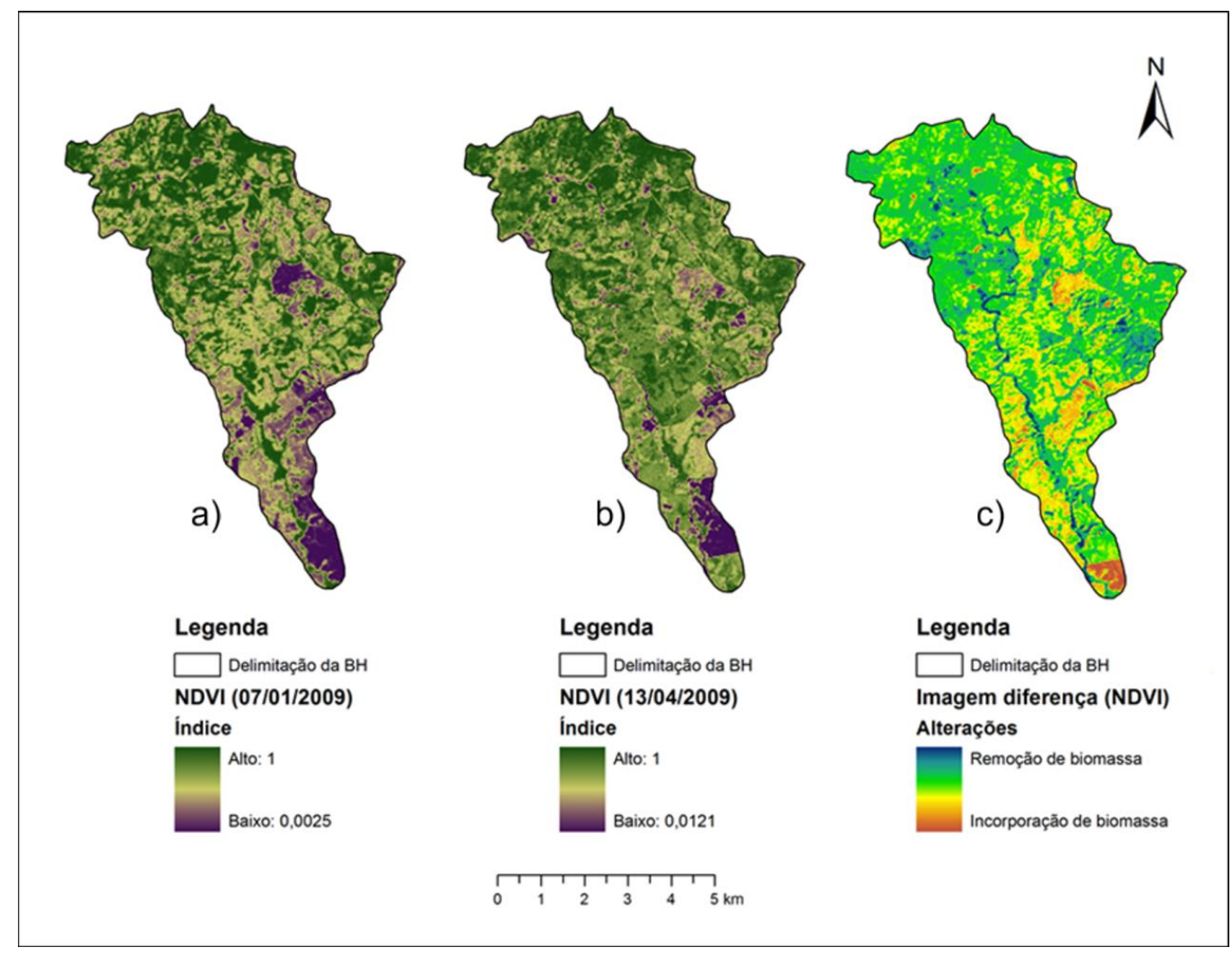

Elaboração: O autor

As alterações ocorridas na zona de vegetação ripária foram decorrentes de reajustes do modelamento natural do leito fluvial. Ao aumentar o recebimento de água e detritos, a morfologia do canal precisou se ajustar para atender aos processos e simultaneamente impor resistência. Este pensamento é corroborado pelo conceito de equilíbrio dinâmico de Chorley (1962), no qual a entrada, circulação e a saída de energia e matéria em um sistema aberto são desencadeadas por meio do ajustamento constante dos elementos e atributos deste sistema aberto (CHRISTOFOLETTI, 1997). Por sua vez o equilíbrio dinâmico é atingido quando as formas passam a representar o resultado contínuo de um ajuste entre o comportamento dos processos e o grau de resistência do material trabalhado (GUERRA; CUNHA, 1994). 
Alterações no canal principal da bacia hidrográfica do Arroio Michaela, Pelotas-RS, decorrentes de um evento pluviométrico extremo do ano de 2009
Henrique Noguez da Cunha Adalberto Koiti Miura Jose Maria Filippini Alba Mauro Ricardo Roxo Nóbrega Adriano Luís Heck Simon

O ajuste representado pelo alargamento do leito do Arroio Michaela, em função da erosão marginal e da remoção da cobertura de vegetação ripária, é a principal consequência do evento extremo de precipitação. Com a enxurrada, a energia cinética da água conduziu o desgaste e o desprendimento do material das margens, alargando o leito.

As Figuras $3 a$ e $3 b$ exibem os índices de vegetação anteriores e posteriores ao evento, respectivamente. Na Figura $3 \mathrm{c}$ as modificações identificadas pelo método da imagem diferença podem ser facilmente verificadas em um contínuo entre duas situações extremas: as áreas de incorporação e de remoção de vegetação. Observam-se na cor azul escuro os locais onde ocorreram remoções de biomassa que estavam presentes na primeira data e ausentes na segunda, principalmente nas áreas próximas ao leito (matas ribeirinhas). O oposto apresenta-se com cor vermelha: as incorporações vegetais presentes na segunda data e que estavam ausentes na primeira data. Nos tons transicionais observa-se o gradiente entre esses dois extremos, sendo que em verde claro e amarelo tem-se uma situação mais específica, na qual a vegetação está presente, que expressa as diferenças sazonais das condições de iluminação e fenologia.

A imagem diferença do NDVI (Figura 3c) permite visualizar as alterações que ocorreram ao longo de toda a bacia durante o período analisado. Destaca-se a mudança no uso das terras em função da dinâmica das atividades agropecuárias e, sobretudo, o resultado do evento climático extremo que marcou a superfície da bacia com a remoção de biomassa e materiais, bem como deposições de detritos e sedimentos ao longo do canal principal e áreas adjacentes.

A interpretação da imagem diferença dos componentes principais e das imagens-fração do modelo linear de mistura espectral mostraram resultados complementares ao do NDVI, minimizando as ambiguidades entre os alvos (Figura 4) e superestimação dos resultados.

Figura 4: a) Cenário anterior ao evento climático; b) Cenário posterior; c) Resultado da classificação sobre imagem diferença do NDVI; d) Resultado sobre imagem diferença do conjunto (NDVI, $1^{\text {a }} \mathrm{CP}$, MLME).

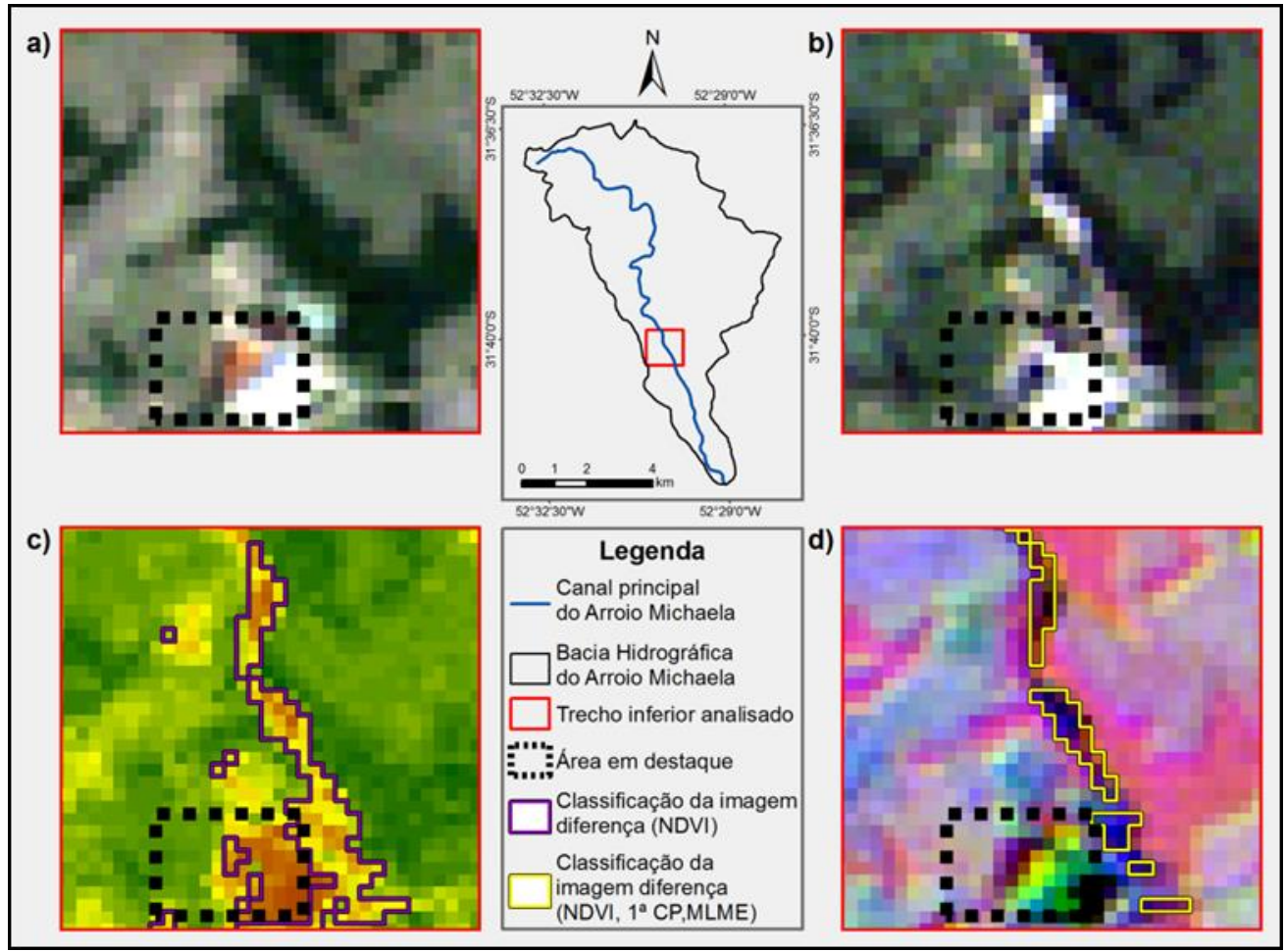

Elaboração: $\mathrm{O}$ autor 
Alterações no canal principal da bacia hidrográfica do Arroio Michaela, Pelotas-RS, decorrentes de um evento pluviométrico extremo do ano de 2009
Henrique Noguez da Cunha Adalberto Koiti Miura Jose Maria Filippini Alba Mauro Ricardo Roxo Nóbrega Adriano Luís Heck Simon

$\mathrm{Na}$ figura 4 (c), corroborando com os estudos de Freitas e Cruz (2003), é possível observar a superestimação do resultado obtido pela classificação da imagem diferença do NDVI, comissionando áreas que estavam sem cobertura vegetal tanto no cenário anterior (Figura 4a) quanto no cenário posterior (Figura 4b), que levaram à tomada de decisão por utilizar as técnicas de diminuição de ruído. Portanto, esse conjunto (Figura 4d) foi utilizado como atributo para classificação supervisionada (Figura 5a, 5b e 5c).

Figura 5: Resultado das classificações supervisionadas da imagem diferença (NDVI, $1^{\text {a }}$ CP e MLME) para a identificação das mudanças ocorridas no compartimento de fundo de vale atrelado à zona de vegetação ripária do canal principal da bacia hidrográfica $(\mathrm{BH})$ do Arroio Michaela.

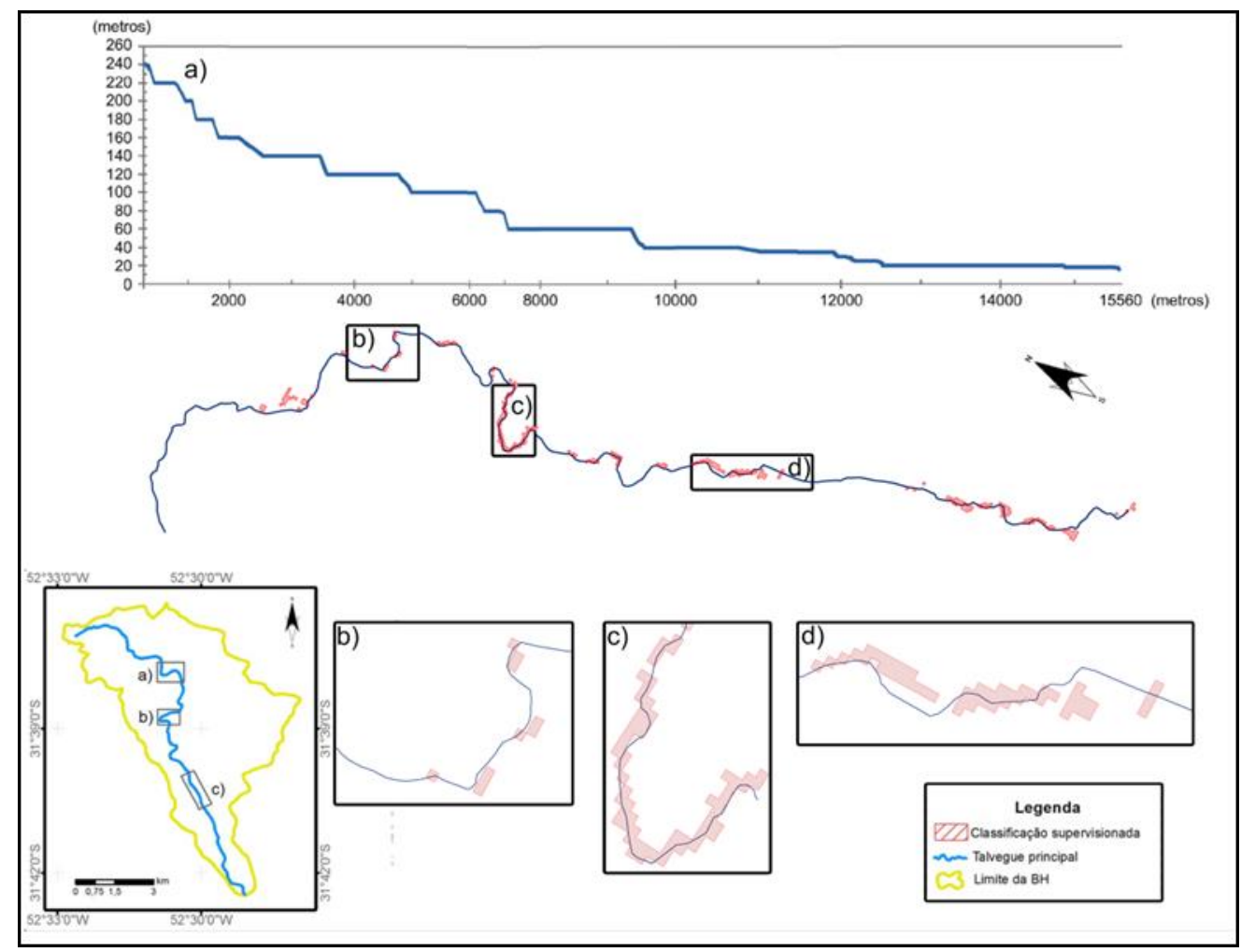

Elaboração: O autor

A quantificação da classe de alterações decorrentes do evento pluviométrico foi de $0,35 \mathrm{~km}^{2}$. O resultado apresentado na Figura 5 indica significativas alterações ocorridas em três setores principais do compartimento de fundo de vale do canal principal, indicados no perfil longitudinal (a) e que se referem à ação de remoção da cobertura vegetal ripária em: (b) segmentos do perfil longitudinal em zona de maiores declividades, (c) segmentos do perfil longitudinal em zonas de maior sinuosidade, e (d) segmentos do perfil longitudinal em zona de menores declividades.

A Figura $5 b$ destaca um trecho situado na área de cabeceiras da $\mathrm{BH}$, com registro fotográfico apresentado na Figura 6, em segmento do perfil longitudinal cujo relevo apresenta altas declividades e altitudes. Esta conjuntura determina a ocorrência de uma área de grande competência fluvial, devido à velocidade de escoamento e a capacidade de transporte de água e materiais, o que é determinante para remoções de biomassa e materiais grosseiros, sobretudo em situações de precipitação excepcional. 
Alterações no canal principal da bacia hidrográfica do Arroio Michaela, Pelotas-RS, decorrentes de um evento pluviométrico extremo do ano de 2009
Henrique Noguez da Cunha Adalberto Koiti Miura Jose Maria Filippini Alba Mauro Ricardo Roxo Nóbrega Adriano Luís Heck Simon

Figura 6: Registro fotográfico das alterações ocorridas no terço superior da $\mathrm{BH}$.

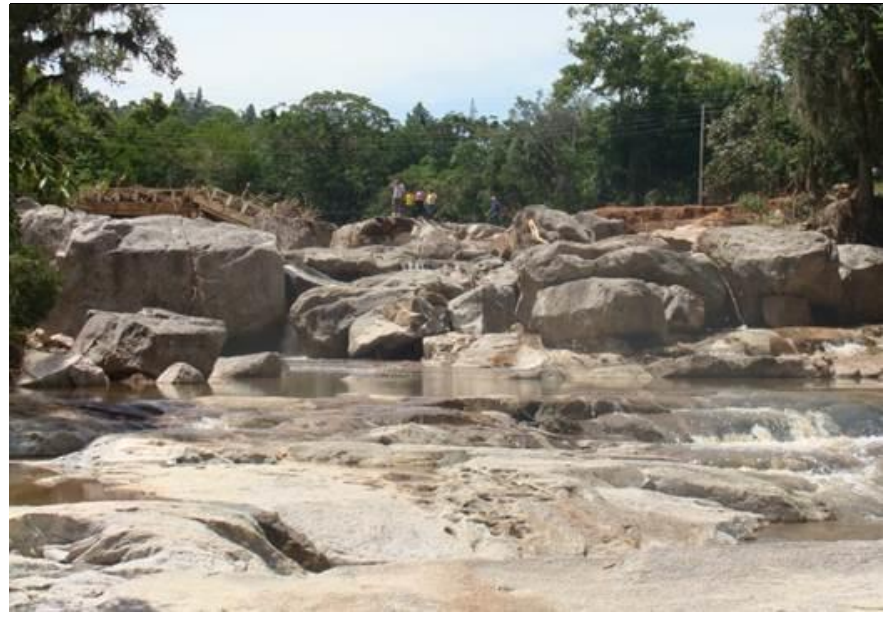

Registro: $\mathrm{O}$ autor

O segmento de canal destacado na Figura $5 \mathrm{c}$, com registro fotográfico na Figura 7 , se situa na porção central da BH, apresentando segundo Cunha (2007), um padrão predominantemente sinuoso atrelado ao controle estrutural, com degradação e agregação de materiais.

Figura 7: Registro fotográfico das modificações ocorridas no segundo terço da BH.

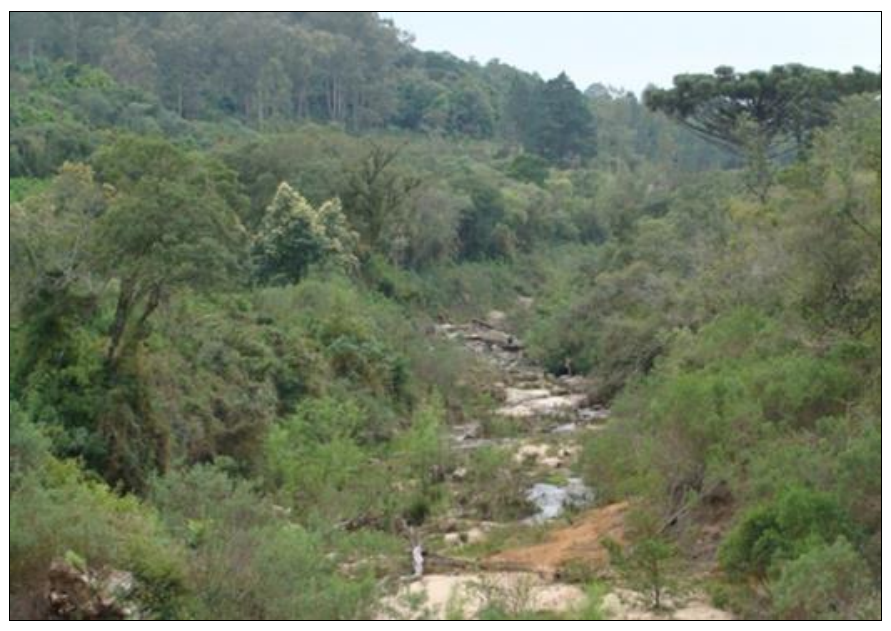

Registro: $\mathrm{O}$ autor

$\mathrm{Na}$ figura $5 \mathrm{~d}$ é possível verificar o trecho mais à jusante do curso principal do Arroio Michaela, onde ocorreu a deposição dos materiais transportados pelos canais fluviais durante o evento extremo. Esta deposição esteve atrelada à diminuição da competência de transporte em virtude da redução da declividade na área de planície, conforme evidencia o registro fotográfico (Figura 8) e o perfil longitudinal (Figura 5a e 5d). Cabe destacar, entretanto, que a deposição ocorrida em um curto espaço de tempo foi responsável por significativas alterações na cobertura vegetal ripária, por meio do soterramento da mesma com material de composição heterogênea, contribuindo para modificações no arranjo espacial do perfil transversal neste segmento do canal fluvial, identificadas a partir da análise da imagem diferença. 
Alterações no canal principal da bacia hidrográfica do Arroio Michaela, Pelotas-RS, decorrentes de um evento pluviométrico extremo do ano de 2009
Henrique Noguez da Cunha Adalberto Koiti Miura Jose Maria Filippini Alba Mauro Ricardo Roxo Nóbrega Adriano Luís Heck Simon

Figura 8: Registro fotográfico das modificações ocorridas no terço inferior da $\mathrm{BH}$.

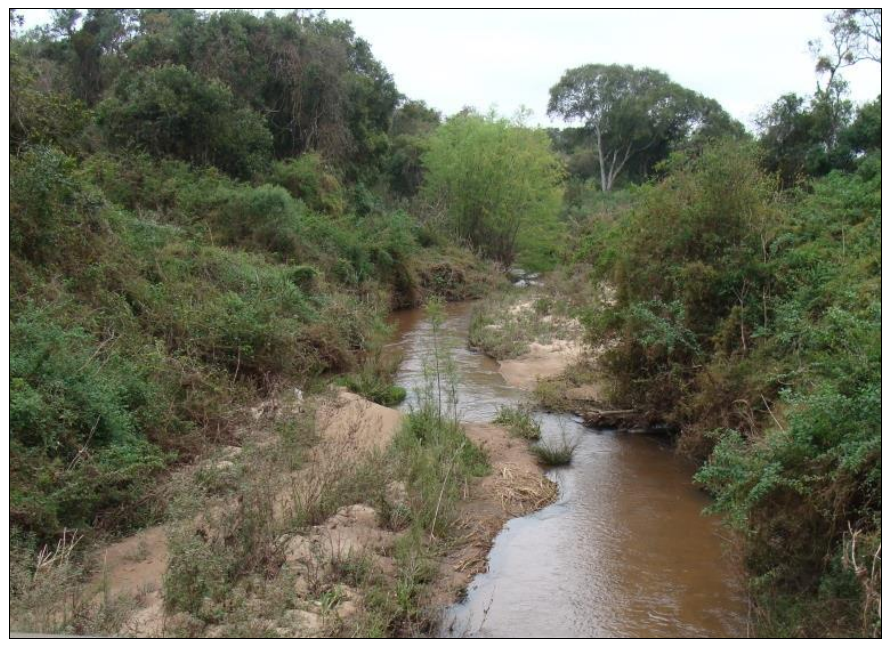

Registro: O autor

A Figura 9 apresenta um conjunto de registros fotográficos obtidos em dois pontos do perfil longitudinal do canal principal da $\mathrm{BH}$ do Arroio Michaela nos momentos anterior e posterior ao evento de precipitação extrema. O primeiro ponto se situa junto à ponte que atravessa o Arroio Michaela, enquanto o segundo ponto se situa à aproximadamente $50 \mathrm{~m}$ de distância à jusante do primeiro ponto. Ambos locais apresentados na Figura 9 compõe as alterações verificadas no segmento de canal fluvial discriminado nas Figuras $5 b$ e 6.

Figura 9: Alterações decorrentes do evento pluviométrico. a) e c): Registros fotográficos anteriores ao evento; b) e d): Registros posteriores ao evento.

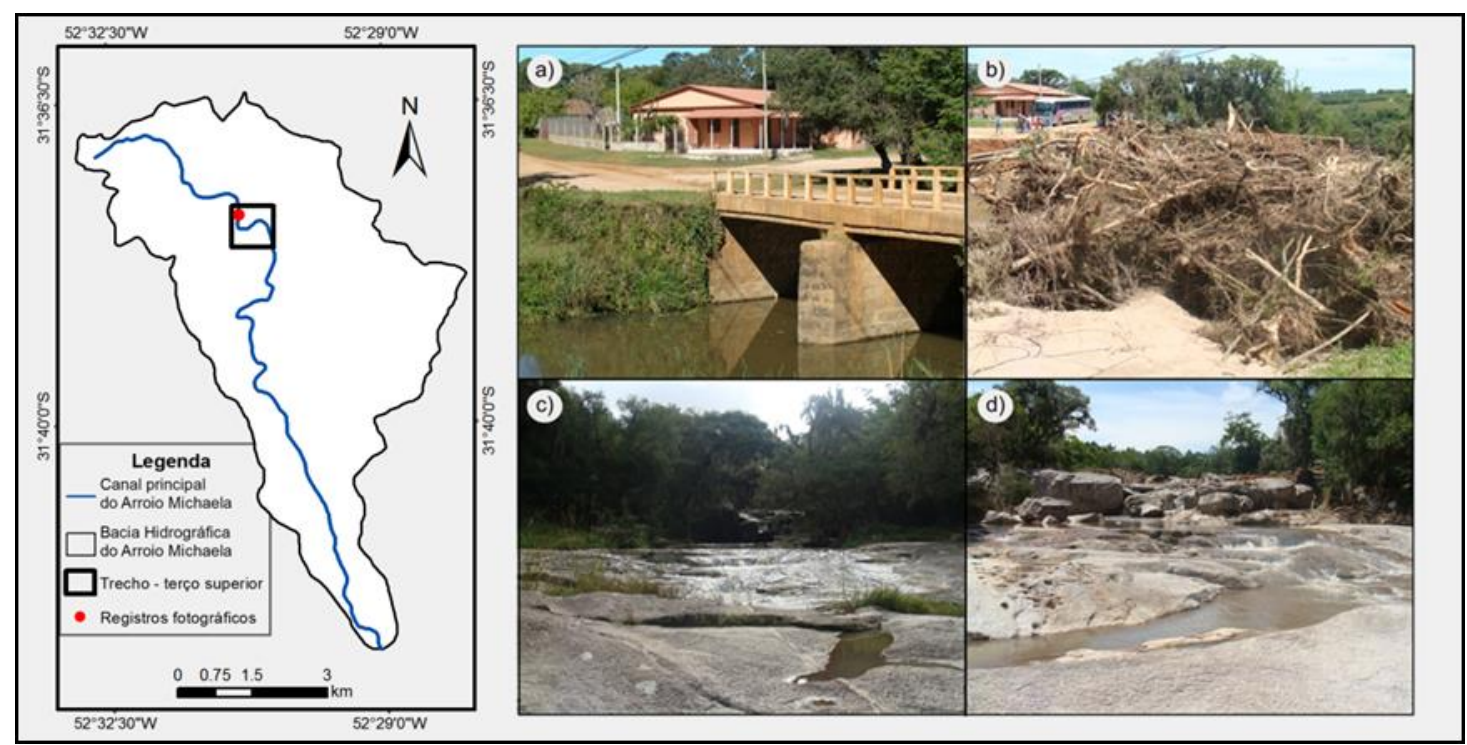

Elaboração: O autor

Na Figuras 9b é possível verificar o acumulo de detritos oriundos do processo de remoção e transporte das cabeceiras da $\mathrm{BH}$ junto à ponte que atravessa o Arroio Michaela em seu alto curso (Figura 9a). Verifica-se ainda o acúmulo de material arenoso no leito do canal e a descaracterização 
Alterações no canal principal da bacia hidrográfica do Arroio Michaela, Pelotas-RS, decorrentes de um evento pluviométrico extremo do ano de 2009
Henrique Noguez da Cunha Adalberto Koiti Miura Jose Maria Filippini Alba Mauro Ricardo Roxo Nóbrega Adriano Luís Heck Simon

do entorno da ponte causada pelo excesso de fluxo e de energia do curso em seu momento máximo de transporte. $\mathrm{O}$ bloqueio da ponte condicionou o represamento de água à montante, induzindo ao desvio do fluxo e à erosão da margem esquerda do Arroio Michaela junto à ponte. O rompimento deste represamento temporário forneceu maior energia e competência fluvial ao novo percurso desencadeando efeitos em cascata nas margens do canal à jusante. Esta situação evidencia a necessidade de entendimento da dinâmica natural dos cursos de água e dos espaços necessários para esta dinâmica, uma vez que a ponto em questão atuou como mecanismo de controle do fluxo em momento de precipitação extrema e acabou condicionando a amplificação dos efeitos do excesso de água e materiais transportados pelo Arroio Michaela.

Nas Figuras 9c e 9d é possível compreender os efeitos do aumento do fluxo de água e materiais no leito do Arroio Michaela durante o evento extremo logo à jusante da ponte: parte da mata ciliar foi removida e a linha de queda de água existente foi completamente exposta, com movimentação de alguns blocos rolados de grande porte devido à energia da corrente. A remoção da cobertura vegetal ripária foi intensificada pelo aumento da energia e capacidade de remoção e transporte resultantes do desbloqueio da barreia de detritos vegetais à montante, exemplificado na figura 9b. Esta remoção da cobertura vegetal marginal ao curso de água pode ser verificada na Figura $9 \mathrm{~b}$.

Dessa forma, tanto o volume escoado como a velocidade da enxurrada foram decorrentes da intensidade, duração e frequência desse evento extremo de precipitação pluviométrica, intensificado pela morfometria da $\mathrm{BH}$, que apresenta um relevo relativamente íngreme nas cabeceiras (Figura 5a).

Dentre os aspectos morfométricos da $\mathrm{BH}$, o perfil longitudinal do talvegue de $220 \mathrm{~m}$, a forma da bacia alongada em leque com fator de forma de 0,15 , o coeficiente de compacidade de 1,738 e sinuosidade do canal principal de 1,36, favoreceram a velocidade de escoamento de águas durante essa precipitação volumosa, facilitando os processos físicos sobre as margens (CUNHA, et al. 2012).

\section{CONSIDERAÇÕES FINAIS}

A BH do Arroio Michaela, apesar de seu pequeno tamanho $\left(37,2 \mathrm{~km}^{2}\right)$, desempenhou um importante papel durante o evento pluviométrico extremo de 2009 ao drenar um grande volume de água ao Arroio Fragata. Como consequência, o leito principal da $\mathrm{BH}$ apresentou inúmeras alterações, com remoção de materiais nas áreas mais à montante (terço superior), desvio resultante de barramentos por infraestruturas, deposição e assoreamento em áreas sinuosas e/ou menos íngremes mais à jusante (terços médio e inferior) e ampliação/exposição do leito fluvial ocasionado, sobretudo pelas características altimétricas do perfil longitudinal perante a grande entrada de água desse evento extremo de precipitação.

O método de detecção de alterações utilizado, imagem diferença, revela-se simples, de fácil implementação e análise. A adoção do índice de vegetação, isolado, não cumpriu com o objetivo, já o conjunto do NDVI com a primeira componente principal e imagens-fração potencializaram a classificação digital viabilizando, de forma refinada, a identificação e quantificação das áreas alteradas, o que a recomenda para trabalhos / estudos similares.

Por fim, deve-se pensar em atribuir a esta metodologia uma importância às ações de planejamento do processo de ocupação por parte das atividades agrícolas, da instalação de infraestrutura publica e da construção de demais obras de engenharia em bacias hidrográficas, pois esta análise permitiu entender o comportamento da rede de drenagem em situações extremas, indicando as áreas com maior tendência à alterações durante estes eventos.

\section{REFERÊNCIAS}

ABDON, M. D. M.; VILA DA SILVA, J. D. S.; SOUZA, Í. D. M.; ROMON, V. T.; RAMPAZZO, J.; FERRARI, D. L. Desmatamento no bioma Pantanal até o ano 2002: relações com a fitofisionomia e limites municipais. Revista Brasileira de Cartografia, v. 59, n. 1, 2009. 
Alterações no canal principal da bacia hidrográfica do Arroio Michaela, Pelotas-RS, decorrentes de um evento pluviométrico extremo do ano de 2009
Henrique Noguez da Cunha Adalberto Koiti Miura Jose Maria Filippini Alba Mauro Ricardo Roxo Nóbrega Adriano Luís Heck Simon

ADAMS, J. B.; SABOL, D. E., KAPOS, V.; ALMEIDA-FILHO, R.; ROBERTS, D. A.; SMITH, M. O.; GILLESPIE, A. R. Classification of multispectral images based on fractions of endmembers: Application to land-cover change in the Brazilian Amazon, Remote Sensing of Environment, v. 52, n. 2, p. 137-154, 1995. https://doi.org/10.1016/0034-4257(94)00098-8

ANABOR, V. Análise descritiva dos sistemas convectivos de escala meso- $\alpha$ através das imagens de satélite GOES - 8. 2004. 78 p. Dissertação (Mestrado em Sensoriamento Remoto). Curso de Pós-Graduação em Sensoriamento Remoto, Centro Estadual de Pesquisas em Sensoriamento Remoto e Meteorologia, Universidade Federal do Rio Grande do Sul. Porto Alegre, 2004.

BARBIERI, D. W.; FERREIRA, C. C.; SAITO, S. M.; SAUSEN, T. M.; HANSEN, M. A. F. Relação entre os desastres naturais e as anomalias de precipitação para a região Sul do Brasil. In: Anais XIV Simpósio Brasileiro de Sensoriamento Remoto, Natal, Brasil, 25-30 abril, INPE, p. 3527-3534, 2009.

BATESON, A.; CURTISS, B. A method for manual endmember selection and spectral unmixing, Remote Sensing of Environment, v. 55, n. 3, p. 229-243, 1996. https://doi.org/10.1016/S00344257(95)00177-8

BRASIL. Ministério das Minas e Energia. Secretaria Geral. Programa de Integração Regional. RADAMBRASIL. Levantamento de Recursos Naturais. Folha SH.22 Porto Alegre e parte das folhas SH.21 Uruguaiana e SI 22 Lagoa Mirim. Rio de Janeiro, 1986. v. 33.

CHANDER, G., MARKHAM, B.L., HELDER, D.L..Summary of Current Radiometric Calibration Coefficients for Landsat MSS, TM, ETM+, and EO-1 ALI Sensors. Remote Sensing of Environment, v. 113, n. 5, p. 893-903, 2009. https://doi.org/10.1016/j.rse.2009.01.007

CHAVEZ JR., P.S. An improved dark-object subtraction technique for atmospheric scattering correction of multiespectral data. Remote Sensing of Environment, v. 24, n. 9, p. 459-479, set. 1988. https://doi.org/10.1016/0034-4257(88)90019-3

CHORLEY, R. J. Geomorphology and general systems theory. Washington, DC: US Government Printing Office, 1962. https://doi.org/10.3133/pp500B

CHRISTOFOLETTI, A. Análise de Sistemas em Geografia. São Paulo: Hucitec, 1979.106p.

CUNHA, H.N. da; PRANKE, L.; MIURA, A.K. Delimitação e caracterização morfométrica fluvial da bacia de drenagem do Arroio Micaela, Pelotas/ RS. Congresso de Iniciação Científica, 21, Pelotas/RS, 20-23 de Nov. 2012. Anais... Pelotas: UFPEL, 2012. 1 CD-ROM.

CUNHA, S. B. Geomorfologia Fluvial. In: Guerra, A. J, T.; Cunha. S.B. Geomorfologia: uma atualização de bases e conceitos. Rio de Janeiro: Bertrand Brasil, 2007. 7, p. 211-252.

DELANEY, P. J. V. Fisiografia e Geologia de Superfície da Planície Costeira do Rio grande do Sul. Boletim da Escola de Geologia da Universidade Federal do Rio Grande do Sul, Porto Alegre, $\mathrm{n}$. 6, p. 1 - 105, 1965. Publicação especial.

DREW, D. Man-environmental processes. George Allen \& Unwin, Londres, 135 p, 1983.

EMBRAPA CLIMA TEMPERADO. Laboratório de Agrometeorologia. Disponível em: $<$ http://www.cpact.embrapa.br/agromet/>. Acesso em: Julho de 2013.

ESRI - ENVIROMENTAL SYSTEMS RESEARCH INSTITUTE. ArcGIS Desktop 9.3 Redlands (CA), 2008.1 CD-ROM.

FREITAS, S. R.; CRUZ, C. B. M.. Índices de vegetação na caracterização de fragmentos de Mata Atlântica no Estado do RJ. Anais... XI Simpósio Brasileiro de Sensoriamento Remoto, INPE, Sao José dos Campos, p. 2737-2744, 2003.

GONG, P., 1993, Change detection using principal component analysis and fuzzy set theory.

Canadian Journal of Remote Sensing, v. 19, n.1, p. 22-29, 1993.

https://doi.org/10.1080/07038992.1993.10855147 
Alterações no canal principal da bacia hidrográfica do Arroio Michaela, Pelotas-RS, decorrentes de um evento pluviométrico extremo do ano de 2009
Henrique Noguez da Cunha Adalberto Koiti Miura Jose Maria Filippini Alba Mauro Ricardo Roxo Nóbrega Adriano Luís Heck Simon

GUERRA, A. J. T. Processos Erosivos nas Encostas. In: GUERRA, A. J. T. E CUNHA, S. B. Geomorfologia: Uma Atualização de Bases e Conceitos. Rio de Janeiro: Bertrand Brasil. p. 149-209, 1994.

HASENACK, H.; WEBER, E. (org.) Base cartográfica vetorial continua do Rio Grande do Sul escala 1:50.000. Porto Alegre: UFRGS/Centro de Ecologia.2010.1 DVD-ROM (Série Geoprocessamento, 3)

IPCC - INTERGOVERNMENTAL PANEL ON CLIMATE CHANGE. Managing the Risks of Extreme Events and Disasters to Advance Climate Change Adaptation. Special Report of the Intergovernmental Panel on Climate Change. Cambridge: Cambridge University Press, 2012. 582 p.

JENSEN, John R. Remote sensing of the environment: An earth resource perspective 2/e. Pearson Education India, 2009.

JOHNSON, R.A.; WICHERN, D.W. Applied multivariate statistical analysis. New Jersey: PrenticeHall, 816p.1998.

LEICA - GEOSYSTEMS GIS \& MAPPING. Erdas Imagine 8.7. Atlanta: Leica Geosystems GIS \& Mapping, 2003. 1 CD-ROM.

LU, D.; MAUSEL, P.; BRONDÍZIO, E.; MORAN, E. Change detection techniques.International Journal of Remote Sensing, v. 25, n. 12, p. 2365-2407. 2004.

https://doi.org/10.1080/0143116031000139863

MACHADO, L. A. T.; ROSSOW, W. B.; GUEDES, R. L.; WALKER, A. W. Life cycle variations of Mesoscale Convective Systems over the Americas. Monthly Weather Review, v. 126, n. 6, p. 16301654, 1998. https://doi.org/10.1175/1520-0493(1998)126<1630:LCVOMC>2.0.CO;2

MARENGO, J.A.; SCHAEFFER, R.; PINTO, H.S.; ZEE, D.M.W. Mudanças climáticas e eventos extremos no Brasil. Rio de Janeiro: FBDS, 2009.

MIURA, A.K. Identificação e quantificação de áreas de Formação Pioneira de Influência Fluvial do litoral centro-sul do Estado do Paraná, por meio de técnicas de sensoriamento remoto. 1999. $119 \mathrm{p}$. Dissertação (Mestrado em Engenharia Floresta).Curso de Pós Graduação em Engenharia Florestal Setor de Ciências Agrárias, Universidade Federal do Paraná, UFPR, Curitiba,1999, $119 p$.

MUCHONEY, D. M., HAACK, B. N. Change detection for monitoring forest defoliation.

Photogrammetric Engineering and Remote Sensing, v. 60, n. 10, p. 1243- 1251. 1994.

PHILIPP, R. P et al. O Magmatismo Granítico Neoproterozóicodo Batólito Pelotas no Sul do Brasil: novos dados e revisão da geocronologia regional. Revista Brasileira de Geociências, São Paulo, v. 32, n. 2, p. 277 - 290, 2002. https://doi.org/10.25249/0375-7536.2002322277290

ROBERTS, D. A.; NUMATA, I.; HOLMES, K.; BATISTA, G.; KRUG, T.; MONTEIRO, A.; CHADWICK, $\mathrm{O}$. A. Large area mapping of land-cover change in Rondônia using multitemporal spectral mixture analysis and decision tree classifiers. Journal of Geophysical Research, v. 107, n. D20, p. 40.001 40.017, 2002.

ROUSE, J. W.; HAAS, R. H.; SCHELL, J. A.; DEERING, D. W. Monitoring vegetation systems in the Great Plains with ERTS. In: ERTS-1 Symposium, 3., 10-14 December, Washington, DC.

Proceedings... Washington, NASA SP-351, p. 309-317, 1973.

SAITO, S. M.; FERREIRA, C. C.; SAUSEN, T. M.; HANSEN, M. A. F.; MARCELINO, I. P. V. O. Sistematização de ocorrências de desastres naturais na região Sul do Brasil. Anais... XIV Simpósio Brasileiro de Sensoriamento Remoto, Natal, Brasil, 25-30 abril 2009, INPE, p. 2333-2339.

SALDANHA; C. B.; COLLISCHONN, W.; MARQUES, M. O Evento de Chuva Intensa de Janeiro de 2009 Sobre a Região de Pelotas-RS. Revista Brasileira de Recursos Hídricos, v.17, n.2; p. 255265, 2012. https://doi.org/10.21168/rbrh.v17n2.p255-265

SCHNEIDER, T. Cidade: Chuva torrencial provoca destruição, mortes e deixa Pelotas ilhada do restante do Estado. Diário Popular, v. 120, 2009. 
Alterações no canal principal da bacia hidrográfica do Arroio Michaela, Pelotas-RS, decorrentes de um evento pluviométrico extremo do ano de 2009
Henrique Noguez da Cunha Adalberto Koiti Miura Jose Maria Filippini Alba Mauro Ricardo Roxo Nóbrega Adriano Luís Heck Simon

SHIMABUKURO, Y. E; Batista, G. T.; Mello, E. M. K.; Moreira, J. C. Duarte, V. Using shade fraction image segmentation to evaluate deforestation in Landsat Thematic Mapper of the Amazon Region. International Journal of Remote Sensing, v. 19, n. 3, p. 535-541. 1998. https://doi.org/10.1080/014311698216152

SHIMABUKURO, Y.E.; Smith, J.A. The least-squares mixing models to generate fraction images derived from remote sensing multispectral data. IEEE Transactions on Geoscience and Remote Sensing. v. 29, p. 16-20, 1991. https://doi.org/10.1109/36.103288

SONG, C.; WOODCOCK, C.E.; SETO, K. C., LENNEY M., P., MACOMBER, S., A. Classification and change detection using Landsat TM data: when and how to correct atmospheric effects. Remote Sensing of Environment, v. 75, n. 2.p. 230- 244, 2001. https://doi.org/10.1016/S00344257(00)00169-3

STRAHLER, A.H. The use of prior probabilities in maximum likelihood classification of remotely sensed data.Remote Sensing of Environment , v.10,n.2, p. 135-163, 1980. https://doi.org/10.1016/0034-4257(80)90011-5

THIAM, A.K., Geographic Information Systems and Remote Sensing Methods for Assessing and Monitoring Land Degradation in the Sahel Region: The case of South Mauritania. 1997, 490p. Tese (Doutoradoemgeografia), Department of Geography,Clark University, Worcester, Massachussets, USA. 1997.

TUCKER, C.J. Red and photographic infrared linear combinations for monitoring vegetation. Remote Sensing of Environment.v.8, n.2, p.127-150, 1979. https://doi.org/10.1016/0034-4257(79)90013-0

TUCKER, C.J.; NEWCOMB, W.W.; LOS, S.O.; PRINCE, S.D. Mean and inter-annual variation of growing-season normalized difference vegetation index for the Sahel 1981-1989. International Journal of Remote Sensing, v. 12, p.1133-1135, 1991. https://doi.org/10.1080/01431169108929717

USGS (United States Geological Survey). Landsat Program. Disponível em: <http://glovis.usgs.gov/>. Acesso em: 03 jan. 2012.

VENTURIERI, A.; FIGUEIREDO, R. O.; WATRIN, O. S.; MARKEWITZ, D. Utilização de imagens Landsat e CBERS na avaliação da mudança do uso e cobertura da terra e seus reflexos na qualidade da água em microbacia hidrográfica do município de Paragominas, Pará. Anais...Simpósio Brasileiro de Sensoriamento Remoto, v. 12, n. 2005, p. 1127-1134, 2005.

Recebido em: 31/10/2017

Aceito para publicação em: 09/05/2018 